\title{
Extremal Disturbance Analysis for Dynamical Systems with Uncertain Input
}

Walter D. Pilkey

wdp@virginia.edu University of Virginia

Dep. of Mechanical and Aerospace Eng. 22904-4746 Charlottesville, VA. USA

Dmitry V. Balandin

balandin@pmk.unn.runnet.ru Nizhny Novgorod State University Dep. of Comp. Mathematics and Cybernetics 603950 Nizhny Novgorod. Russia

Nikolai N. Bolotnik bolotnik@ipmnet.ru Institute for Problems in Mechanics of the Russian Academy of Sciences 119526 Moscow. Russia

\section{Sergey V. Purtsezov}

pusv@virginia.edu University of Virginia

Dep. of Mechanical and Aerospace Eng. 22904-4746 Charlottesville, VA. USA
The philosophy of the extremal disturbance analysis for dynamical systems with uncertain inputs is described. This analysis involves solving optimal control problems in which the time histories of the inputs (external disturbances) are regarded as the control functions and a response measure of the system serves as the performance index. The performance index should be maximized or minimized over the disturbances within a prescribed class. Often this class is specified by lower and upper bounds (a corridor) between which the values of the disturbance must lie. The maximization and minimization problems are referred to as the worst disturbance and best disturbance problems, respectively. The solutions of these problems provide the extreme values between which the response measure lies for any disturbance from the specified class. The extremal disturbance analysis is important, in particular, when designing standards for testing devices for the protection of fragile objects from impact loads. This approach is illustrated for a singledegree-of-freedom system that can be regarded as a simplified model of the equipment for sled tests of automobile restraint systems.

Keywords: Uncertain input, worst disturbance, best disturbance, impact isolation, sled tests

\section{Introduction}

There are numerous structural dynamics problems where the loading is not fully defined. Typically, such problems are treated as stochastic problems using the theory of probability (Hanss, 2005; Iyenger, 1972). This paper discusses an alternative deterministic approach in which the available information on the loading is utilized to calculate bounds on possible responses of the structure. The lower bound would be the least maximum response that can occur. The corresponding loading is referred to as the best disturbance. The upper bound is the greatest possible peak response and the corresponding loading is the worst disturbance (Koivuniemi, 1966; O'Hara, 1973). The response of the structure to any other loading falling within the prescribed loading bounds will lie between the extreme values of the response.

One example occurs if an explosive environment is characterized in terms of a total impulse. This is sufficient information to determine what must happen to some equipment, e.g., military equipment, and the maximum response of this equipment.

Other example occurs in the testing of safety devices for crashing vehicles. For instance, tie-down systems for wheelchairs are tested on high-speed sleds for which the crash pulse must adhere to standards that are defined in terms of an envelope in which the crash pulse must lie (Kang and Pilkey, 1998). An even more common example occurs in the sled testing of child seats, where the sled pulse must lie within prescribed time-varying bounds (Crandall et al., 1996). The proposed scheme provides upper and lower bounds for critical responses. These responses can be used to help gauge whether the prescribed pulse corridor is too tight or too broad

Presented at XI DINAME - International Symposium on Dynamic Problems of Mechanics, February 28th - March 4th, 2005, Ouro Preto. MG. Brazil.

Paper accepted: June, 2005. Technical Editors: J.R.F. Arruda and D.A. Rade. to provide effective standards. Thus, the procedure here develops a technology to study the sensitivity of dynamic responses to incompletely prescribed test conditions.

We choose to use the sled testing of child seats as an example of using extremal disturbance analysis to illustrate the principles of this sensitivity technology.

\section{Nomenclature}

$A, B, C, D=$ coordinates of the vertices of the trapezoid, defined by Eq. (28), (time, acceleration), ( $\mathrm{s}, \mathrm{m} / \mathrm{s}^{2}$ )

$a=$ height of the trapezoid, defined by Eq. (27), acceleration, $\mathrm{m} / \mathrm{s}^{2}$

$c=$ damping coefficient of the restraint system, damping coefficient, $\mathrm{kg} / \mathrm{s}$

$F$ = force applied to the sled, force, $N$

$f=$ force applied to the dummy by the restraint system, force, N

$g(t)=$ impulse response of the system, $s$

$h=$ time between samples, time, $s$

$i$ = sample number, dimensionless

$j=$ design variable number, dimensionless

$J$ = peak magnitude of the absolute acceleration of the dummy, acceleration, $\mathrm{m} / \mathrm{s}^{2}$

$K(t)=$ function, defined by Eq. (21), $1 / s$

$k=$ stiffness coefficient of the restraint system, stiffness coefficient, $\mathrm{kg} / \mathrm{s}^{2}$

$L=$ number of samples, dimensionless

$M=$ mass of the sled, mass, $\mathrm{kg}$

$m=$ mass of the dummy, mass, $\mathrm{kg}$

$N=$ number of design variables, dimensionless

$p$ = parameter defined by Eq. (22), dimensionless 
$R=$ sensitivity of the peak force transmitted to the dummy relative to the variation in the crash pulse, dimensionless

$R_{d}=$ prescribed value of the sensitivity of the peak force transmitted to the dummy relative to the variation in the crash pulse, dimensionless

$T=$ duration of the crash pulse, time, $s$

$t$ = time, $s$

$u=$ absolute acceleration of the dummy with minus sign, acceleration, $\mathrm{m} / \mathrm{s}^{2}$

$W=$ class of the design variables $w$, acceleration, $\mathrm{m} / \mathrm{s}^{2}$

$w=$ absolute acceleration of the sled with minus sign treated as a design variable, acceleration, $\mathrm{m} / \mathrm{s}^{2}$

$w_{-}(t)=$ lower bound of the design variable $w$, acceleration, $\mathrm{m} / \mathrm{s}^{2}$

$w_{+}(t)=$ upper bound of the design variable $w$, acceleration, $\mathrm{m} / \mathrm{s}^{2}$

$x=$ displacement of the dummy relative to the sled, displacement, $m$

$z=$ displacement of the sled relative to the fixed frame, displacement, $m$

\section{Greek Symbols}

$\Delta v=$ velocity change during the crash, velocity, $\mathrm{m} / \mathrm{s}$

$\Delta_{-} v=$ lower bound of the velocity change during the crash, velocity, $\mathrm{m} / \mathrm{s}$

$\Delta_{+} v=$ upper bound of the velocity change during the crash, velocity, $\mathrm{m} / \mathrm{s}$

$\delta(t)=$ Dirac delta function, dimensionless

$\varsigma=$ fraction of critical damping, dimensionless

$\lambda=$ similarity factor, dimensionless

$\lambda_{*}=$ similarity factor equal to 1.14 , dimensionless

$\mu=$ reduced mass of the system, defined by Eq. (2), mass, $\mathrm{kg}$

$\tau=$ variable of integration, time, $\mathrm{s}$

$\tau_{1-4}=$ parameters of the trapezoid, defined by Eq. (27), time, $\mathrm{s}$

$\psi(t)=$ function, representing a trapezoid and defined by

Eq. (27), acceleration, $\mathrm{m} / \mathrm{s}^{2}$

$\omega_{n}=$ angular undamped natural frequency of the system, angular frequency, $\mathrm{rad} / \mathrm{s}$

$\omega_{D}=$ angular damped natural frequency of the system, angular frequency, $\mathrm{rad} / \mathrm{s}$

\section{Subscripts}

$i$ relative to sample number

$j$ relative to design variable number

0 relative to the optimal solution of the best disturbance problem

- relative to the lower bound of the design variable $w$

+ relative to the upper bound of the design variable $w$

\section{Superscripts}

0 relative to the optimal solution of the worst disturbance problem

+ relative to the upper bound of the design variable $w$

\section{Basic Concepts of the Best and Worst Disturbance Analyses; Statement of the Optimal Control Problems}

A sled test is often employed to investigate protective characteristics of safety devices. For example, automobile restraint systems can be studied with sled tests. In particular, consider a sled test for an automobile restraint that restricts the motion of an occupant to prevent a violent contact with components inside the car during a crash. Typically, the test sled equipment consists of a highspeed sled with a mock-up of the car interior, an occupant seat, a restraint system, e.g., seat belt, and a dummy as the occupant. The car mock-up with interior and seatbelts are rigidly attached to the sled. The sled, dummy, and the seat belts are equipped with sensors that measure accelerations, displacements, strains, forces, and other characteristics of the response of the system. The sled is accelerated to a prescribed velocity and then is subject to a deceleration pulse simulating the crash impact. The various measured responses can be used to form injury criteria that can be compared with established tolerance levels.

To fix ideas, we consider a simplified model of the testing equipment in which the sled and the dummy are regarded as rigid bodies (Fig. 1). The sled moves along a horizontal straight line relative to a fixed (inertial) reference frame and the dummy moves relative to the sled along the same line. Let $z$ denote the displacement (coordinate) of the sled relative to the fixed frame, $x$ the displacement (coordinate) of the dummy relative to the sled, $M$ the mass of the sled, $m$ the mass of the dummy, $F$ the force applied to the sled during the crash simulation, and $f$ the force applied to the dummy by the restraint system. The mass of the seat belts is neglected as compared with the masses of the sled and the dummy. The force $f$ is assumed to be a function of the parameters of the relative motion of the dummy, the relative coordinate $x$ and the relative velocity $\dot{x}$, i.e., $f=f(x, \dot{x})$. This function takes into account the elastic and dissipative properties of the seatbelts. The motion of this mechanical system is governed by the simultaneous equations

$$
M \ddot{z}=F-f, \quad m(\ddot{x}+\ddot{z})=f,
$$

which represent Newton's second law for the sled and the dummy, respectively. The dummy is acted upon only by the restraint system force $f$, whereas the sled is acted upon by the impact force $F$ and the force $-f$ as the seat belt interacts with the sled.

Solve the system of Eq. (1) for $\ddot{x}$ and $\ddot{z}$ to obtain

$$
\mu \ddot{x}=f(x, \dot{x})-F \frac{m}{M+m}, M \ddot{z}=F-f(x, \dot{x}), \quad \mu=\frac{M m}{M+m} .
$$

The quantity $\mu$ is called the reduced mass of the system. In the system of Eq. (2), the equation governing the motion of the dummy relative to the sled is independent of the equation of motion of the sled relative to the fixed frame. If one is interested only in the relative motion of the dummy, and the impact force is a known function of time, i.e., $F=F(t)$, only the first relation of (2) is used. In the theory of impact isolation, the impact disturbance specified as the force pulse acting on the base (the sled) is referred to as the dynamic disturbance.

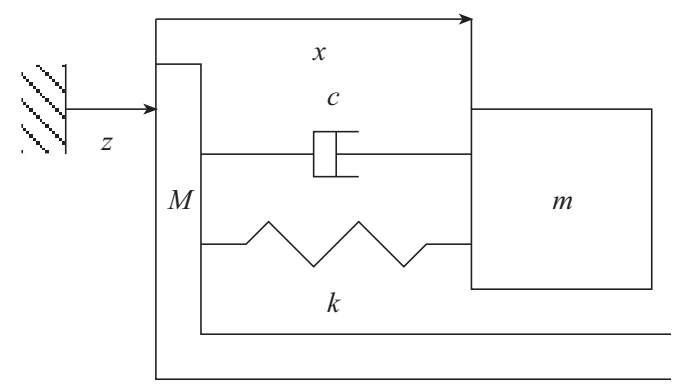

Figure 1. Single-degree-of-freedom model. 
In a number of cases, it is reasonable to assume that the impact disturbance is characterized by the acceleration of the base, rather than by the force applied to it. For instance, for the sled tests, the impact deceleration of the sled is measured by accelerometers. Then the relative motion of the body to be protected (the dummy) is governed by the second relation of Eq. (1) where $\ddot{z}(t)$ is treated as a known function of time. The impact disturbance specified as the base acceleration pulse is referred to as the kinematic disturbance.

For both types of disturbances, the equation of the relative motion of the body to be protected can be represented in a unified form as

$$
\ddot{x}+u(x, \dot{x})=w(t)
$$

where $u(x, \dot{x})=-f(x, \dot{x}) / \mu, \quad w(t)=-F(t) / M$ for the dynamic disturbance and $u(x, \dot{x})=-f(x, \dot{x}) / m, \quad w(t)=-\ddot{z}(t)$ for the kinematic disturbance. In the theory of impact isolation, the function $u(x, \dot{x})$ is frequently called the characteristic of the impact isolator. As a rule, Eq. (3) is subjected to zero initial conditions

$$
x(0)=0, \dot{x}(0)=0 .
$$

These conditions imply that the object to be protected does not move relative to the base until the impact pulse has been applied to the base.

To compare the disturbances in terms of the response of the object to be protected, one should have a numerical measure of this response. We select

$$
J(w)=\max _{t}|u(x(t), \dot{x}(t))|
$$

where $x(t)$ stands for the solution of the differential equation (3) subject to the initial conditions of Eq. (4) for a given $w=w(t)$. The quantity $J$ characterizes the peak magnitude of the force transmitted to the dummy by the restraint system. (Actually, the peak force is equal to $\mu J$ or $m J$ for the case of the dynamic or kinematic disturbance, respectively.) The measure $J(w)$ is a functional of the disturbance, depending on the entire time history of $w$.

Consider now the statement of the worst and best disturbance problems for the system of Eqs. (3) and (4) with the response measure of Eq. (5). Let the disturbances belong to a prescribed class $W$, i.e., $w \in W$. Note that $W$ is a set of functions rather than a set of values of these functions. In terms of the sled test, the class $W$ can characterize a set of disturbances that are representative of the test, taking into account the inevitable inaccuracy in the reproduction of the desired deceleration pulse.

Usually, the class of representative disturbances involves a "corridor" or "envelope" (Fig. 2) in which these disturbances must lie, i.e.,

$$
W=\left\{w(t): w_{-}(t) \leq w(t) \leq w_{+}(t), t \in[0, T]\right\},
$$

where $w_{-}(t)$ and $w_{+}(t)$ are prescribed functions that define the lower and upper bounds of the corridor and $T$ is a fixed time. In addition, the velocity of the sled should change by a certain value during the crash pulse time $T$. In the case of the kinematic disturbance, this condition has the form

$$
\Delta v=\int_{0}^{T} w(\tau) d \tau
$$

where $\Delta v$ is a specified positive quantity. Usually, the quantity $\Delta v$ is prescribed with an allowance for an error in the measurement of the velocity of the sled,

$$
\Delta_{-} v \leq \Delta v \leq \Delta_{+} v,
$$

where the interval $\left[\Delta_{-} v, \Delta_{+} v\right]$ characterizes the uncertainty in the measurement of the velocity decrease. Since $\Delta v$ is defined as the integral of $w$, the inequalities of Eq. (8) impose additional constraints on the deceleration pulse time history. Therefore, the total class to which the allowed deceleration pulses must belong is defined by

$$
W=\left\{w(t): w_{-}(t) \leq w(t) \leq w_{+}(t), \Delta_{-} v \leq \int_{0}^{T} w(\tau) d \tau \leq \Delta_{+} v\right\} .
$$

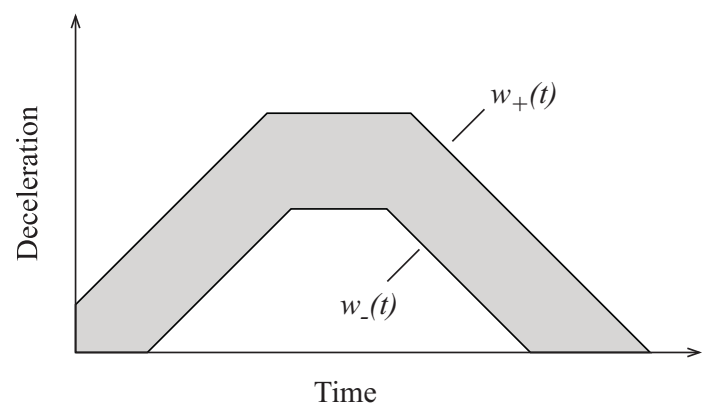

Figure 2. Corridor in which an input pulse w(t) must lie.

The worst disturbance problem is that of the determination of the upper bound of the functional $J(w)$ for the disturbances of the class $W$.

Problem 1 (Worst Disturbance Problem). For the system governed by Eqs. (3) and (4), find the disturbance function $w^{0}(t)$ belonging to the class $W$ such that

$$
J\left(w^{0}\right)=\max _{w \in W} J(w)
$$

The best disturbance problem is that of the determination of the lower bound of the functional $J(w)$ for the disturbances of the class $W$.

Problem 2 (Best Disturbance Problem). For the system governed by Eqs. (3) and (4), find the disturbance function $w_{0}(t)$ belonging to the class $W$ such that

$$
J\left(w_{0}\right)=\min _{w \in W} J(w) .
$$

From the mathematical point of view Problems 1 and 2 are optimal control problems with a maximum type functional, since the performance index in these problems is the maximum of the absolute value of the force transmitted to the dummy over the impact response time.

The worst and best disturbance analyses enable one to investigate the sensitivity of the results of the sled test to the variation of the impact pulse within the corridor prescribed by the standards for such tests. If the difference between the upper and lower bounds is large, the test may have an undesirably low value of the peak force transmitted to the dummy due to the occurrence of the disturbance close to the best one. Such a test can be deceptive when assessing the quality of the restraint system and, hence, is illdesigned. In this case, the standards for the defining allowable impact pulses should be revised.

Sometimes, the sensitivity of the peak force transmitted to the dummy relative to the variation in the impact pulse is characterized by the ratio 


$$
R=J\left(w^{0}\right) / J\left(w_{0}\right)
$$

\section{Restraint System with Linear Characteristics; Numerical Solution of the Optimal Control Problems}

Let the restraint system have linear elastic and damping properties, i.e.,

$$
f(x, \dot{x})=-c \dot{x}-k x,
$$

where $c$ and $k$ are the damping and stiffness coefficients, respectively. For an impact disturbance of the kinematic type, Eq. (3) becomes

$$
\ddot{x}+2 \varsigma \omega_{n} \dot{x}+\omega_{n}^{2} x=w(t),
$$

where $\omega_{n}$ is the natural frequency of the system and $\varsigma$ is the damping ratio,

$$
\omega_{n}=(k / m)^{1 / 2}, \quad \varsigma=c /\left(2(k m)^{1 / 2}\right) .
$$

The criterion $J$ of Eq. (5) (the peak magnitude of the occupant's absolute acceleration) becomes

$$
J(w)=\max _{t}\left|2 \varsigma \omega_{n} \dot{x}(t)+\omega_{n}^{2} x(t)\right| .
$$

Even for the linear system of Eq. (14), the solution of the best and worst disturbance problems (Problems 1 and 2) requires numerical methods. To this end, the continuous-time formulation of these problems is replaced by a discrete-time approximation. The time axis is discretized with step size $h$. On the time intervals $(i-1) h \leq t<i h, \quad i=1,2, \ldots$, the function $w(t)$ is assumed to be constant:

$$
w(t)=\left\{\begin{array}{l}
w_{i}, \text { if }(i-1) h<T \\
0, \text { if }(i-1) h \geq T
\end{array}, \quad(i-1) h \leq t<i h\right.
$$

The constant parameters $w_{i}, \quad i=1, \ldots, N$, play the role of design variables when solving the optimization problems. The number of these design variables is defined as follows:

$$
N=\left\{\begin{array}{l}
T / h, \text { if }\{T / h\}=0 \\
{[T / h]+1, \text { if }\{T / h\} \neq 0}
\end{array},\right.
$$

where the square brackets and the braces denote the integer and fractional parts, respectively, of the expression enclosed.

The solution of Eq. (14) subject to the initial conditions of Eq. (4) has the convolution form

$$
x(t)=\int_{0}^{t} g(t-\tau) w(\tau) d \tau, \quad \dot{x}(t)=\int_{0}^{t} \dot{g}(t-\tau) w(\tau) d \tau,
$$

where

$$
g(t)=\left\{\begin{array}{l}
\omega_{D}^{-1} \exp \left(-\zeta \omega_{n} t\right) \sin \omega_{D} t, \text { if } \varsigma<1, \\
t \exp \left(-\varsigma \omega_{n} t\right), \text { if } \varsigma=1, \\
\omega_{D}^{-1} \exp \left(-\zeta \omega_{n} t\right) \sinh \omega_{D} t, \text { if } \varsigma>1,
\end{array} \omega_{D}=\omega_{n}\left|1-\varsigma^{2}\right|^{1 / 2} .\right.
$$

The function $g(t)$ is referred to as the impulse response function (fundamental solution) for Eq. (14). It satisfies the differential equation of (14) with $w(t)=\delta(t)$, where $\delta(t)$ is the Dirac delta function, subject to the initial conditions $x(0)=0$ and $\dot{x}(t)=0$. This function can also be defined as the solution of Eq. (14) with zero right-hand side ( $w(t) \equiv 0)$, subject to the initial conditions $x(0)=0$ and $\dot{x}(0)=1$.

Substitute the relations of Eq. (20) into Eq. (16) to obtain

$$
J=\max _{t}\left|\int_{0}^{t} K(t-\tau) w(\tau) d \tau\right|, \quad K(t)=2 \varsigma \omega_{n} \dot{g}(t)+\omega_{n}^{2} g(t) .
$$

The discrete-time approximation of the response measure of Eq. (21) has the form

$$
\begin{gathered}
J(w)=\max _{i \in[1: L]}\left|\sum_{j=1}^{\min \{i, N\}} p_{i j} w_{j}\right|, \\
p_{i j}=\int_{(j-1) h}^{j h} K(i h-\tau) d \tau, \quad j \leq i, \quad w=\left[w_{1}, \ldots, w_{N}\right],
\end{gathered}
$$

where $L$ is the number of discrete intervals on the time axis on which the response to the impact pulse is to be calculated. To obtain reliable results, it is necessary to consider the response on the time interval substantially exceeding that of the crash pulse. Since the crash pulse has a rather short duration, the response measure can attain its maximum after the disturbance has ceased to act. For this reason, the number of time instants at which the response is measured should exceed the number of the discretization points in the crash pulse interval $[0, T]$ and, hence, $L>N$.

The criterion of Eq. (22) is a function of $N$ variables $w=\left[w_{1}, \ldots, w_{N}\right]$. The constraints of Eq. (9) are discretized as follows:

$$
w_{-}(i h) \leq w_{i} \leq w_{+}(i h), i \in[1: N] ; \quad \Delta_{-} v \leq h \sum_{j=1}^{N} w_{j} \leq \Delta_{+} v .
$$

In the discrete-time formulation, the worst-disturbance problem is reduced to the maximization of the function of Eq. (22) and the best-disturbance problem to the minimization of this function, subject to the constraints of Eq. (23). These constraints are linear relative to the design variables $w_{1}, \ldots, w_{N}$, and the function to be maximized or minimized is a maximum of the absolute values of linear functions of these variables. In this case, the constrained minimization (maximization) problem can be reduced to that of the linear programming, which substantially facilitates the solution, since there are rapidly converging reliable linear programming algorithms available in most optimization software. For a more detailed description of the solution of optimization problems similar to Problems 1 and 2 on the basis of the linear programming, see Balandin, Bolotnik, and Pilkey (2001).

\section{Numerical Example}

Assume the damping and stiffness coefficients of the restraint systems are:

$$
c=200 \frac{\mathrm{Ns}}{\mathrm{m}}, \quad k=10^{5} \frac{\mathrm{N}}{\mathrm{m}}, \quad m=23 \mathrm{~kg},
$$


which are reasonably realistic for restraint systems. The mass of the occupant of $23 \mathrm{~kg}$ corresponds to the 6-year-old child dummy. Figures 3 to 5 demonstrate the results of the solution of Problems 1 and 2 for the sled deceleration pulse corridor shown in Fig. 3. The corridor corresponds to the standard of NHTSA (1979) that specifies test conditions for child restraints. The uncertainty interval of Eq. (8) for the sled velocity change is defined as

$$
\begin{aligned}
& 45.1 \mathrm{~km} / \mathrm{h} \leq \Delta v \leq 48.3 \mathrm{~km} / \mathrm{h} \\
& (28 \mathrm{mph} \leq \Delta v \leq 30 \mathrm{mph}),
\end{aligned}
$$

Figure 3 shows the time histories for the best and worst deceleration pulses. The shaded area in Fig. 3 represents the corridor in which deceleration pulses must lie. If there were no constraints such as of Eq. (25) on the velocity change, both the best and worst disturbance pulses would have switched between the lower and upper bounds of the corridor. This follows since the solution of a linear programming problem always lies on the boundary of the domain constraining the design variables. From the time histories shown in Fig. 3 it is apparent that both the best disturbance and worst disturbance pulses lie within the walls of the corridor on some time intervals. Therefore, the velocity change assumes one of the boundary values prescribed by Eq. (25), specifically, $\Delta v=45.1 \mathrm{~km} / \mathrm{h}$ for the best disturbance and $\Delta v=48.3 \mathrm{~km} / \mathrm{h}$ for the worst disturbance.

Figure 4 depicts the time history of the force applied to the dummy by the seat belt in the case of the worst (solid curve) and best (dashed curve) disturbances. It is apparent that in both cases the peak magnitude of the force is attained before the end of the disturbance. This is because the duration of the pulse is close to the undamped vibration period of the system. In accordance with figs. 3 , the duration of the deceleration pulse lies between 0.075 and $0.09 \mathrm{~s}$, while the vibration period for the parameters of Eq. (24) is 0.096s.

The time histories of the displacement of the dummy for the worst and best disturbances are shown in Fig. 5. In fact, the curves of Fig. 5 repeat those of Fig. 4 scaled by the stiffness coefficient $\left(10^{5} \mathrm{~N} / \mathrm{m}\right)$. This is due to the fact that the damping does not have much effect during the time interval under consideration. The damping ratio $\varsigma$ of Eq. (15) calculated for the system with the parameters of Eq. (24) equals $0.066 \mathrm{~s}^{-1}$ and, accordingly, the characteristic time of the damping equals $15.2 \mathrm{~s}$, which substantially exceeds the undamped vibration period.

The sensitivity ratio of Eq. (12) for the forces transmitted to the dummy is

$$
R=10.2 / 7.6=1.34 \text {. }
$$

This reasonably low value would probably be acceptable in practice.

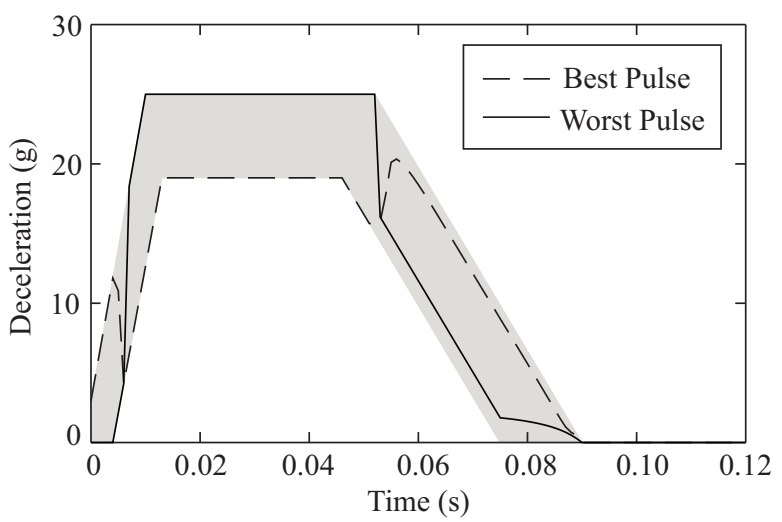

Figure 3. Best and worst disturbances.

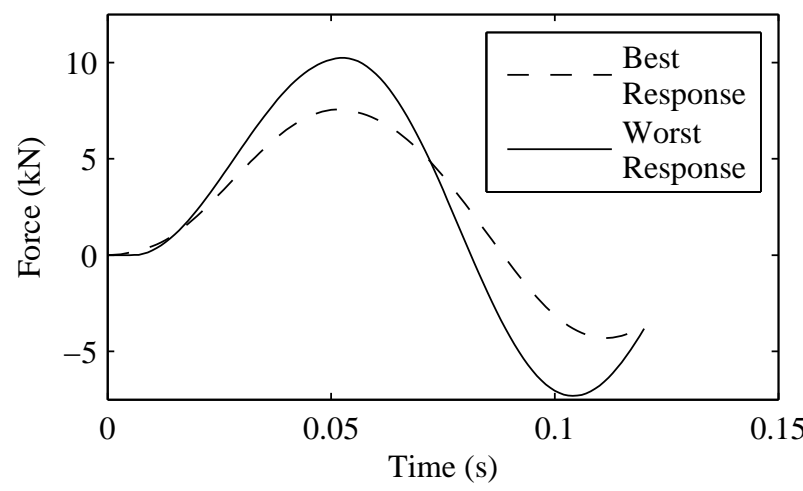

Figure 4. Force applied to the dummy by the seat belt.

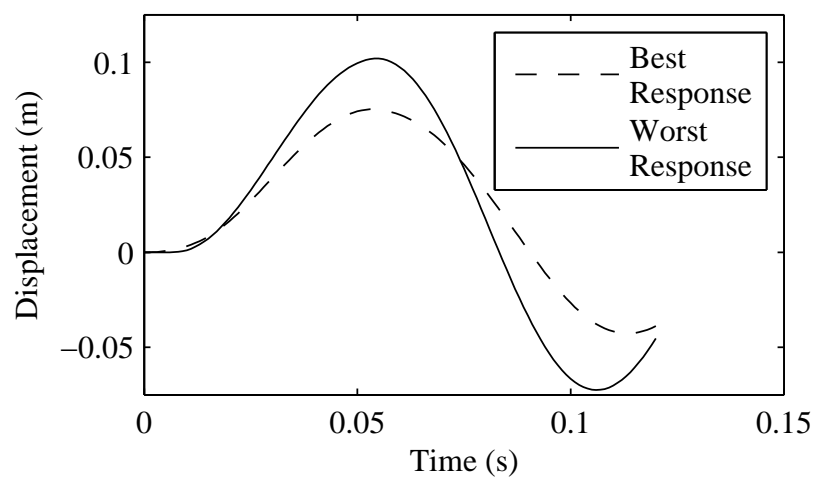

Figure 5. Displacement of the dummy relative to the sled.

\section{Rational Design of Sled Test Standards}

In the previous section, we have illustrated the application of the extremal disturbance analysis to check the sensitivity of the response measure (the force transmitted to the occupant) to the variation of the sled deceleration pulse within a prescribed corridor. This concept suggests a technique for the rational design of such a corridor so that the sensitivity ratio does not exceed an allowed value. Consider an example.

Let the walls of the corridor have a shape of a trapezoid $A B C D$ shown in Fig. 6. A generic trapezoid can be represented analytically by the piecewise linear function 


$$
\psi(t)=\left\{\begin{array}{l}
0, \quad t \in\left(-\infty, \tau_{1}\right) \cap[0, \infty), \\
a\left(t-\tau_{1}\right) / \tau_{2}, \quad t \in\left[\tau_{1}, \tau_{1}+\tau_{2}\right) \cap[0, \infty), \\
a, \quad t \in\left[\tau_{1}+\tau_{2}, \tau_{1}+\tau_{3}\right) \cap[0, \infty), \\
a\left(t-\tau_{1}-\tau_{4}\right) /\left(\tau_{3}-\tau_{4}\right), \quad t \in\left[\tau_{1}+\tau_{3}, \tau_{1}+\tau_{4}\right) \cap[0, \infty), \\
0, \quad t \geq \tau_{1}+\tau_{4}
\end{array}\right.
$$

The coordinates of the vertices of this trapezoid on the timeacceleration plane are given by

$$
\begin{array}{ll}
A=\left(\tau_{1}, 0\right), & B=\left(\tau_{1}+\tau_{2}, a\right), \\
C=\left(\tau_{1}+\tau_{3}, a\right), & D=\left(\tau_{1}+\tau_{4}, 0\right) .
\end{array}
$$

In terms of the geometry of the trapezoid, $\tau_{4}$ and $\tau_{3}-\tau_{2}$ are the lengths of the lower and upper bases, respectively, $a$ is the height, $\left(\tau_{2}^{2}+a^{2}\right)^{1 / 2}$ and $\left(\left(\tau_{4}-\tau_{3}\right)^{2}+a^{2}\right)^{1 / 2}$ are the lengths of the lateral sides $A B$ and $C D$, respectively. The parameter $\tau_{1}$ characterizes the shift of the trapezoid along the time axis.

The walls (the upper and lower bounds) of the corridor for sled tests shown in Fig. 3 have the shape of trapezoids with

$$
\begin{aligned}
& \tau_{1}=4 \mathrm{~ms}, \quad \tau_{2}=9 \mathrm{~ms}, \quad \tau_{3}=42 \mathrm{~ms}, \\
& \tau_{4}=71 \mathrm{~ms}, \quad a=19 \mathrm{~g}
\end{aligned}
$$

for the lower bound and

$$
\begin{aligned}
& \tau_{1}=-1 \mathrm{~ms}, \quad \tau_{2}=11 \mathrm{~ms}, \quad \tau_{3}=53 \mathrm{~ms}, \\
& \tau_{4}=91 \mathrm{~ms}, \quad a=25 \mathrm{~g}
\end{aligned}
$$

for the upper bound. With a high degree of accuracy, the trapezoid of the upper bound is similar to the trapezoid of the lower bound with the similarity factor close to 1.3.

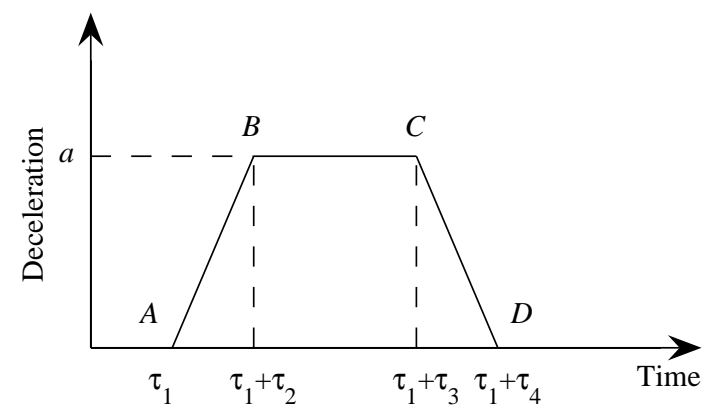

Figure 6. A trapezoidal bound for the force applied to the dummy by the seat belt.

We will confine ourselves to the case where the functions $w_{-}(t)$ and $w_{+}(t)$ of Eq. (6) are represented by similar trapezoids. Let the lower bound be fixed and let $w_{-}(t)=\psi(t)$, where $\psi(t)$ is defined by Eq. (27). Let the upper bound be described by the function

$$
w_{+}(t)=\left\{\begin{array}{l}
0, \quad t \in\left(-\infty, \tau_{1}^{+}\right) \cap[0, \infty), \\
\frac{a\left(t-\tau_{1}^{+}\right)}{\tau_{2}}, \quad t \in\left[\tau_{1}^{+}, \tau_{1}^{+}+\lambda \tau_{2}\right) \cap[0, \infty), \\
\lambda a, \quad t \in\left[\tau_{1}^{+}+\lambda \tau_{2}, \tau_{1}^{+}+\lambda \tau_{3}\right) \cap[0, \infty), \\
\frac{a\left(t-\tau_{1}^{+}-\lambda \tau_{4}\right)}{\left(\tau_{3}-\tau_{4}\right)}, \quad t \in\left[\tau_{1}^{+}+\lambda \tau_{3}, \tau_{1}^{+}+\lambda \tau_{4}\right) \cap[0, \infty), \\
0, \quad t \geq \tau_{1}^{+}+\lambda \tau_{4},
\end{array}\right.
$$

where $\lambda$ and $\tau_{1}^{+}$are the parameters of the upper bound, the similarity factor and the time coordinate of the left-hand bottom vertex (point $A$ ) of the respective trapezoid. For the curve $w_{+}(t)$ of Eq. (31) to lie above the curve $w_{-}(t)=\psi(t)$ of Eq. (27) it is necessary that

$$
\lambda \geq 1, \quad \tau_{1}+(1-\lambda) \tau_{4} \leq \tau_{1}^{+} \leq \tau_{1}
$$

It is apparent that for $\lambda=1$, the lower and upper bounds of the corridor match one another.

By varying the parameters $\lambda$ and $\tau_{1}^{+}$within the domain of Eq. (32) one can assure that the ratio $R$ of Eq. (12) does not exceed a prescribed value. The variation can be organized in various ways. For example, one can constrain the parameter $\tau_{1}^{+}$to lie in the middle of the interval allowed for this parameter by Eq. (32), i.e.,

$$
\tau_{1}^{+}=\tau_{1}+(1-\lambda) \tau_{4} / 2
$$

In this case, the variation is performed with respect to only one parameter, $\lambda$. As $\lambda$ increases, the corridor becomes wider and, therefore, the best-disturbance response measure decreases while the worst-disturbance response measure increases. Accordingly, the sensitivity ratio $R$ is a monotonically increasing function of $\lambda$. The search for the maximum $\lambda$ that assures that the quantity $R$ does not exceed the prescribed value $R_{d}$ is reduced to the solution of the equation $R(\lambda)=R_{d}$. This equation can be solved by various methods, for example, by the interval bisection method. For each trial $\lambda$, one should solve the best disturbance and worst disturbance problems to calculate $R(\lambda)$.

Figure 7 presents the curve $R(\lambda)$, calculated for the corridor defined by Eqs. (27), (29), (31), and (33), completed by the velocity change interval of Eq. (25). The curve becomes more flat as $\lambda$ increases. Note that this curve begins with $\lambda=\lambda \approx 1.14$, rather than with $\lambda=1$, in which case the upper and lower bounds of the crash pulse corridor coincide. This is because of the constraint of Eq. (25) on the velocity change. The lower bound for the velocity change is $45.1 \mathrm{~km} / \mathrm{h}$, whereas the integral of the lower bound of the pulse corridor (the area of the trapezoid representing the respective wall of the corridor) is $34.9 \mathrm{~km} / \mathrm{h}$. Therefore, the pulse of the lower bound is unable to decelerate to a complete stop even for the least crash velocity allowed by the interval of Eq. (25). Moreover, no pulse of the corridor is able to provide a velocity change of $45.1 \mathrm{~km} / \mathrm{h}$ while the area of the upper bound trapezoid is less than this value. This is the case for $\lambda<\lambda_{*}$. For $\lambda=\lambda_{*}$, the area of the upper bound trapezoid is equal to $45.1 \mathrm{~km} / \mathrm{h}$. In this case, the only pulse of the corridor able to decelerate the sled crashing at this velocity to a complete stop is that of the upper bound and, 
accordingly, the worst and best disturbances coincide, i.e., $R\left(\lambda_{*}\right)=1$.

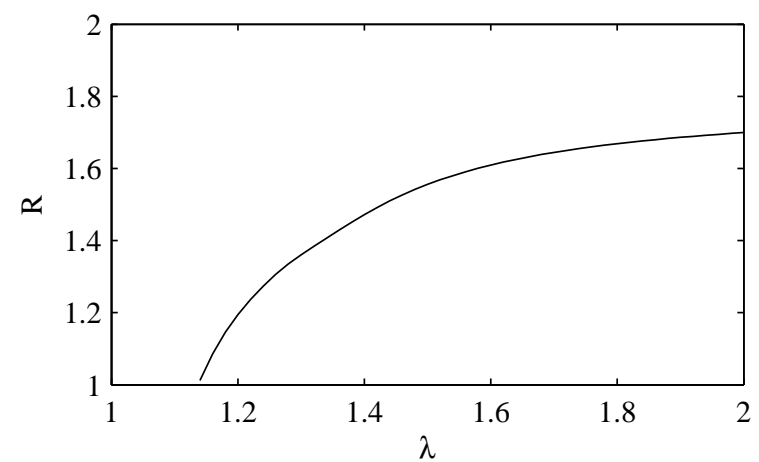

Figure 7. Sensitivity of the peak force transmitted to the dummy relative to the variation in the impact pulse $R$ versus the similarity factor $\lambda$.

\section{Conclusions}

The extremal disturbance analysis enables one to evaluate the sensitivity of the response of a dynamical system to the variation in the external disturbances within a prescribed uncertainty class. To that end one should solve two optimal control problems, the best disturbance problem to calculate the lower bound and the worst disturbance problem to calculate the upper bound of a response measure. The ratio of these bounds can serve as the sensitivity index. The closer this ratio is to unity, the lower the sensitivity. The extremal disturbance analysis is, in particular, important for the validation of standards for impact tests of fragile objects and the equipment for the protection of such objects. Such standards commonly prescribe a corridor for impact pulses to be reproduced on the test facility. The test data are considered to be reliable if the spread in the responses to the input pulses within the corridor is reasonably small. This spread is characterized by the sensitivity ratio resulting from the extremal disturbance analysis. In addition, the extremal disturbance analysis suggests a technique for rational design of a corridor for loading pulses so that the sensitivity ratio does not exceed a prescribed value.

\section{Acknowledgments}

This research was partly supported by NSF (grant BES0302337), NATO (grant CBP.NR.NRCLG 982082), and the Russian Foundation for Basic Research (grants 05-01-00563 and 0401-00222)

\section{References}

Balandin, D.V., Bolotnik, N.N., and Pilkey, W.D., 2001, "Optimal Protection from Impact, Shock and Vibration", Taylor and Francis Publishers, Philadelphia, PA, 437 p.

Crandall, J.R., Pilkey, W.D., Kang, W., and Bass, C.R., 1996, "Sensitivity of Occupant Response Subject to Prescribed Corridors", Journal of Shock and Vibration, Vol. 3, No. 6, pp. 435-450.

Hanss, M., 2005, "Applied Fuzzy Arithmetic - An Introduction with Engineering Applications”, Springer, Berlin, 256 p.

Iyenger, R. N., 1972, "Worst Inputs and a Bound on the Highest Peak Statistics of a Class of Nonlinear Systems", Journal of Sound and Vibration, Vol. 25, No. 1, pp. 29-37.

Kang, W., and Pilkey, W.D, 1998 "Crash Simulations of Wheelchair Occupant Systems in Transport", Journal of Rehabilitation Research and Development, Vol. 35, No. 1, pp. 73-84.

Koivuniemi, A.J., 1966, "Parameter Optimization in Systems Subject to Worst Bounded Disturbance", IEEE Transactions on Automatic Control, Vol. 11, No. 3, pp. 427-433.

National Highway Traffic Safety Administration (NHTSA), 1979 (October, 2000 ed.), "FMVSS 213 Child Restraint Systems", Vol. 49 CFR 571.213 .

O’Hara, G.J., 1973, “Maximization and Minimization of Dynamic Load Factors", Shock and Vibration Bulletin, Vol. 43, Part 2, pp. 119-127. 